\title{
Nodular regenerative hyperplasia, portal vein thrombosis, and avascular hip necrosis due to hyperhomocysteinaemia
}

\author{
O Buchel, T Roskams, B Van Damme, F Nevens, J Pirenne, J Fevery
}

Gut 2005;54:1021-1023. doi: 10.1136/gut.2004.055921

A male patient with portal hypertension, portal vein thrombosis, spontaneous splenorenal shunt formation, and encephalopathy, thought to have post-hepatitis B cirrhosis, is described. His condition deteriorated and necessitated liver transplantation. In the explant liver, nodular regenerative hyperplasia with pronounced vascular lesions both in portal venules and in arterioles was found instead of classical cirrhosis. Two years post-transplant he developed bilateral ischaemic femur head necrosis. The three disorders (portal vein thrombosis, nodular regenerative hyperplasia, and ischaemic hip necrosis) seemed to be due to a common vasculopathy induced by hyperhomocyteinaemia. Genetic studies showed that he carried a mutation in the gene encoding for formation of methylenetetrahydrofolate reductase. Treatment with folic acid combined with pyridoxine (vitamin B6) and cyanocobalamin (vitamin B12) normalised his serum homocysteine levels.

$\mathrm{N}$ odular regenerative hyperplasia of the liver is characterised by the presence of diffuse rather small regenerative nodules in the absence of fibrosis. It is thought to result from occlusion of the terminal branches of hepatic arterioles and portal venules, secondary to endothelial cell damage. ${ }^{1-3}$ It is associated with systemic and vascular disorders such as systemic lupus erythematodes, progressive systemic sclerosis, Sjögren's syndrome, polyarteritis nodosa, rheumatoid arthritis, ${ }^{1245}$ and with coagulopathies such as anticardiolipin and antiphospholipid antibodies or factor $\mathrm{V}$ Leiden mutation. ${ }^{467}$ An association with myeloproliferative and lymphoproliferative disorders has also been described.

We describe a patient with nodular regenerative hyperplasia of the liver and portal vein thrombosis, eventually requiring liver transplantation. This was followed by bilateral avascular necrosis of his femur heads. In looking for coagulopathies, it was demonstrated that this patient had hyperhomocysteinaemia of intermediate grade. Further study documented that he was a heterozygote for the methylenetetrahydrofolate reductase gene C677T mutation. To our knowledge this is the first report of nodular regenerative hyperplasia associated with hyperhomocysteinaemia.

\section{CASE REPORT}

A 51 year old male with mild psoriasis, for which he never received any medication that could induce portal hypertension, was evaluated for possible liver transplantation. He had initially been seen at the haematology clinic for suspected myelodysplasia because of a marked splenomegaly with leuco- and thrombocytopenia (table 1). Further investigations established the presence of an atrophic right liver lobe (atrophy of segments 4 and 5) and a hypertropic caudate and left liver lobe, with portal vein thrombosis and a spontaneous splenorenal shunt. He had pronounced oesophageal varices with a variceal pressure of $20.6 \mathrm{~mm} \mathrm{Hg}$ and a hepatic portal vein pressure gradient of $25 \mathrm{~mm} \mathrm{Hg}$, mild encephalopathy (ammonia levels above $80 \mu \mathrm{M}$; normal values $<50 \mu \mathrm{M}$ ), and osteopenia. He was thought to have post-hepatitis B cirrhosis as he was hepatitis B core antibody positive.

There were no signs of malabsorption and the concentration of tissue transglutaminase-IgA antibody, a serum marker of coeliac disease, was $3.93 \mathrm{U} / \mathrm{ml}$, which is below the cut off value of $7 \mathrm{U} / \mathrm{ml}$ (Genesis, Eden Research Park, Littleport, Cambridgeshire, UK). Because of progressive asthenia, osteopenia, and encephalopathy, he underwent liver transplantation with thrombectomy of the portal vein. The explanted liver did not show cirrhosis but diffuse nodular regenerative hyperplasia with pronounced vascular abnormalities (fig 1). Phlebosclerosis was observed as absence of the portal vein tributary in the smallest ramifications of the portal tracts whereas a small thick walled vein with signs of arterialisation were seen in other tracts together with several arterial branches. In yet other portal tracts, a thrombosed and partly recanalised vein was seen. Ductopenia was present in smaller portal fields. A mild inflammatory infiltrate was present in the parenchyma, as well as large cell dysplasia. Near the hilum of the liver, a thrombosed middle sized artery was observed.

He received a short course of low dose methylprednisolone ( $16 \mathrm{mg}$ for one week, followed by $12 \mathrm{mg} /$ day for three weeks and $8 \mathrm{mg}$ /day tapered over three months), together with tacrolimus and $50 \mathrm{mg}$ of azathioprine as immunosuppression. He did not develop rejection episodes and has suffered no psoriatic lesions since the transplantation. Two years following transplantation, he complained of severe bilateral hip pain. $X$ rays and magnetic resonance imaging documented bilateral avascular hip necrosis, despite the fact that he had only received a tapered doses of corticosteroid therapy for four months. He received a bilateral hip replacement in two operative settings. Histology confirmed the avascular necrosis.

As he expressed a collection of conditions, all indicating a vascular aetiology, we performed a series of tests for thrombophilias, and documented hyperhomocysteinaemia (30.5 $\mu \mathrm{M}$; normal range 7-15). Other thrombophilic factors were within normal limits (table 1). Genetic testing demonstrated a mutation in the gene encoding for formation of methylenetetrahydrofolate reductase. After treatment with folic acid $1 \mathrm{mg}$, pyridoxine $50 \mathrm{mg}$, and cyanocobalamine $500 \mu \mathrm{g} /$ day, his serum homocysteine levels decreased from $30.5 \mu \mathrm{M}$ to an average of $10 \mu \mathrm{M}$.

\section{DISCUSSION}

We have presented a patient, presumed to have post-hepatitis $\mathrm{B}$ cirrhosis with portal vein thrombosis, who underwent liver transplantation for severe hepatic encephalopathy. The explanted liver did not reveal cirrhosis but nodular regenerative hyperplasia. Nodular regenerative hyperplasia of the liver is a rare condition which most often (in 59\% of case reports in the literature ${ }^{2}$ ) presents with stigmata of portal 

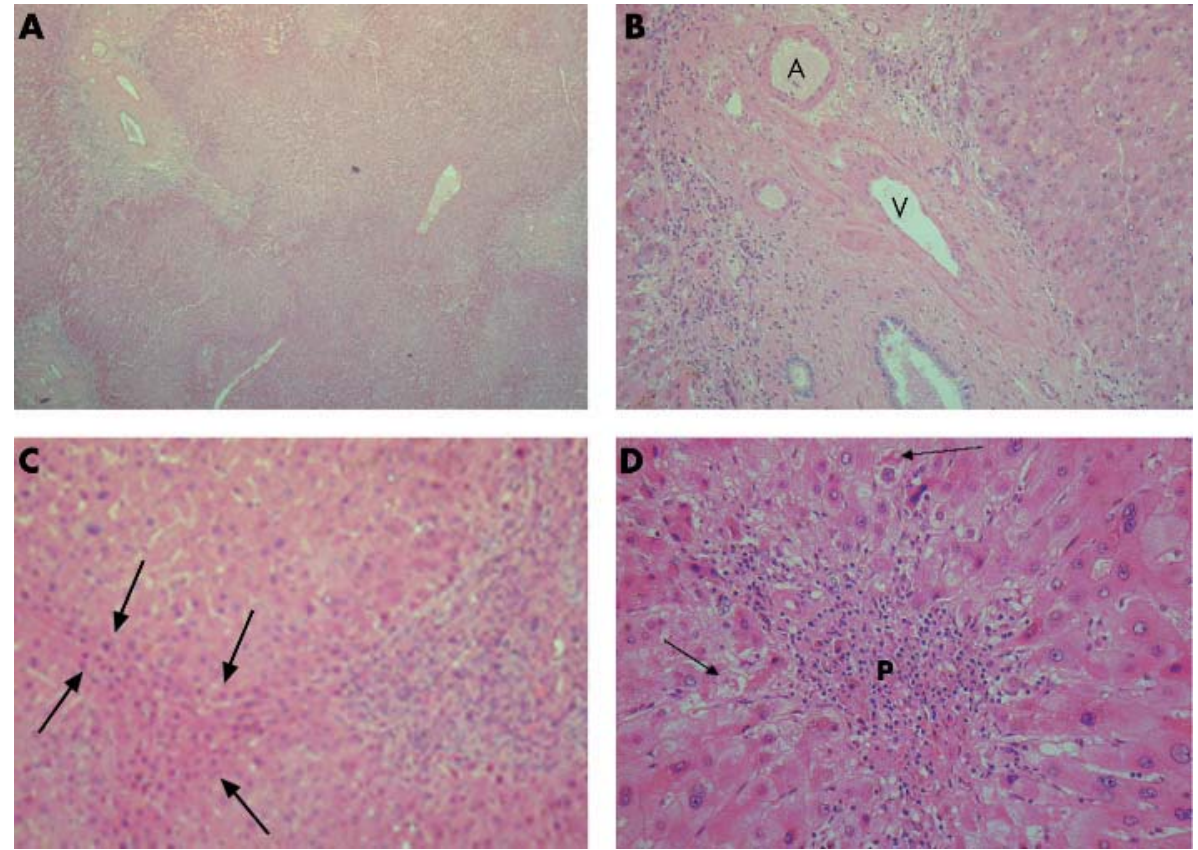

Figure 1 Pathology of the explanted liver. (A) Haematoxylin-eosin stain of a liver section showing nodularity of the parenchyma, without the presence of septa. Portal tracts (for example, upper left corner) show an artery and bile duct of approximately the same size while the portal vein branch is clearly narrowed and its wall is muscularised. (B) Higher magnification of the portal tract from fig 1: the branch of the hepatic artery (A) has the appropriate size while the portal vein branch (V) is narrowed and has a thickened muscularised wall. (C) Nodularity of the parenchyma is due to alternating hypertrophic and atrophic zones. The arrows indicate compressed atrophic muralia. (D) Haematoxylin-eosin stain of a liver section showing a ductopenic portal tract (P). The periportal hepatocytes are characterised by cholate stasis and contain cholestatic Mallory bodies (arrows).

hypertension and its sequelae. The most prevalent disturbance in liver tests is increased alkaline phosphatase and gamma glutamyl transferase levels (in keeping with space occupying lesions) but transaminases may also be raised. A minority of patients are jaundiced or have a raised serum bilirubin. These findings are largely noted in patients who present clinically whereas patients who have nodular

Table 1 Representative laboratory tests before transplantation

\begin{tabular}{|c|c|c|}
\hline & Patient & $\begin{array}{l}\text { Normal } \\
\text { values }\end{array}$ \\
\hline Haemoglobin (g/dl) & 12.1 & $14-18$ \\
\hline Leucocytes $\left(\times 10^{9} / \mathrm{l}\right)$ & 2.2 & $4-10$ \\
\hline Platelets $\left(\times 10^{9} / \mathrm{I}\right)$ & 37 & $150-450$ \\
\hline $\operatorname{ALP}(U / I)$ & 321 & $<270$ \\
\hline AST (U/I) & 62 & $<38$ \\
\hline Bilirubin (mg/dl) & 1.91 & $<1.0$ \\
\hline Ammonia $(\mu \mathrm{mol} / \mathrm{l})$ & 83 & $11-32$ \\
\hline $\lg A(g / l)$ & 6.48 & $0.8-4.53$ \\
\hline $\lg G(g / l)$ & 18.1 & $7.5-15.6$ \\
\hline Folic acid serum ( $\mu \mathrm{g} / \mathrm{l})$ & 5.0 & $2.0-15.0$ \\
\hline Folic acid in red cells $(\mu \mathrm{g} / \mathrm{l})$ & 269 & $200-700$ \\
\hline Prothrombin (s) & 11.4 & $9.5-14$ \\
\hline Activated prothrombin time (s) & 37 & $24-38$ \\
\hline Factor VIII (\%) & 153 & $50-150$ \\
\hline Lupus anticoagulans & Slightly pos & \\
\hline Cardiolipin antibodies (binding index) & 1.0 & $0-2.5$ \\
\hline Protein S (\%) & 85 & $70-150$ \\
\hline Protein C (\%) & 100 & $70-150$ \\
\hline Activated protein $C$ resistance ratio & 2.62 & $2.10-3.10$ \\
\hline Homocysteine $(\mu M)$ & 30.5 & $7-15$ \\
\hline Prothrombin gene mutation & Absent & \\
\hline Factor $\mathrm{V}$ gene mutation & Absent & \\
\hline MTHFR gene C677T mutation & Present & \\
\hline & (heterozygote) & \\
\hline
\end{tabular}

ALP, alkaline phosphatase; AST, aspartate aminotransferase; MTHFR, methylenetetrahydrofolate reductase. regenerative hyperplasia as an incidental autopsy finding have a much lower prevalence of documented clinical and biochemical abnormalities. This is in keeping with the hypothesis that nodular regenerative hyperplasia is the end manifestation of a spectrum of liver diseases with diffuse micronodular transformation as a reflection of progressive degrees of hepatic portal venopathy (occlusion of terminal portal venules), with or without hepatic arterial disorders, depending on the underlying disease. ${ }^{2}$ Hepatic portal venopathy, resulting in ischaemia in affected regions of the liver, is the assumed pathogenetic mechanism, leading to atrophy or apoptosis and then to compensatory hyperplasia and nodule formation in unaffected areas. $^{3}$ When a critical proportion of portal venules are affected, portal hypertension ensues. $^{2}$

Our patient undoubtedly had vascular abnormalities, with combined arterial and portal venous wall thickening, thrombotic phenomena, and absence of portal venules in several tracts. The arterial thrombosis was probably responsible for the biliary features (ductopenia and cholate stasis) as bile ducts depend on an arterial blood supply. He presented clinically with portal hypertension. Nodular regenerative hyperplasia was not our initial diagnosis as he was assumed to have post-hepatitis B cirrhosis at the time of transplantation. This is due to the inherent shortcomings of needle biopsy of the liver and has also been reported elsewhere in the literature. ${ }^{2}$ Our patient also had hepatic encephalopathy, presumably due to the large spontaneous splenorenal shunt, as encephalopathy is not usually observed in patients with nodular regenerative hyperplasia.

Two years after transplantation a diagnosis of bilateral avascular necrosis of the femur heads was established. Avascular necrosis of the femur head has been described in patients receiving corticosteroid therapy after renal transplantation. ${ }^{8}$ It may be related to the number of pulse doses of intravenous methylprednisolone given for acute rejection as well as to the cumulative dose. Avascular necrosis of the 


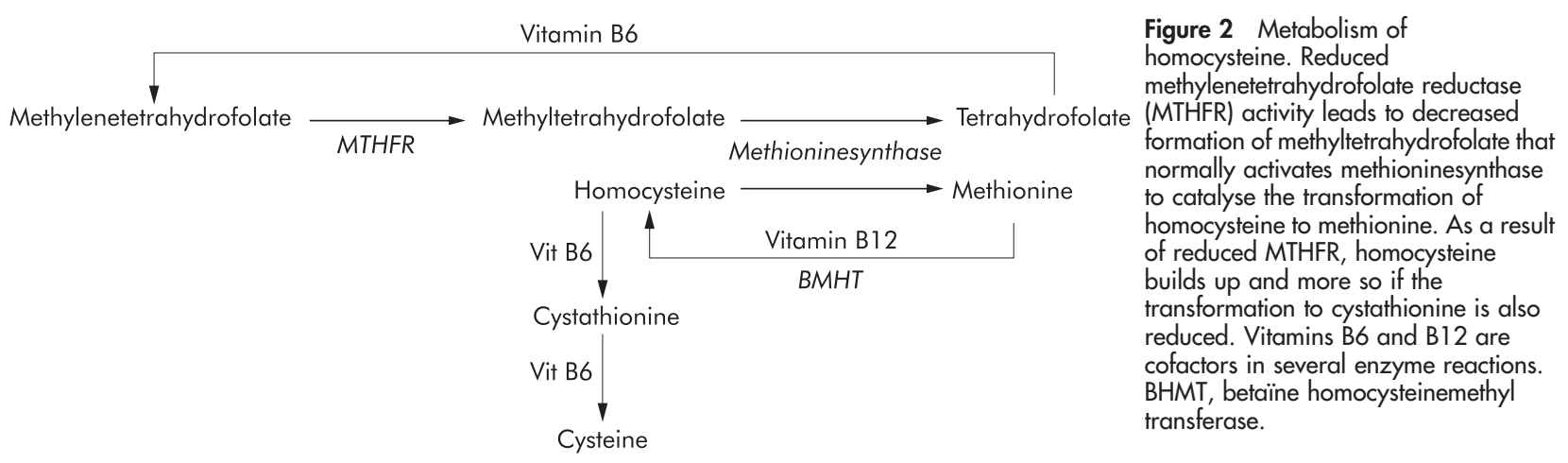

femur head has also been described in patients with psoriasis receiving topical corticosteroid therapy. This however is related to the fact that topical preparations may be applied, in extended courses, to diseased skin, thus facilitating systemic absorption of significant amounts of corticosteroid. ${ }^{9}$ Our patient presented with avascular necrosis of both femur heads two years after cessation of a three month tapered course of corticosteroids. It is therefore very unlikely that the course of steroids given was the cause of the femur head necrosis. Documentation of a markedly raised plasma homocysteine level however provides a potential link between seemingly unrelated disease processes.

Genetic studies revealed that he was heterozygous for the 677 C-T mutation of the methylenetetrahydrofolate reductase gene. This results in substitution at position 222 of alanine to valine in the reductase. This mutated enzyme is thermolabile and has reduced enzyme activity. ${ }^{10}$ The decreased reductase activity leads to enhanced levels of homocysteine, and more so if a low folate state is present as the formation of 5-methyltetrahydrofolate is reduced. The latter is a cosubstrate for remethylation of homocysteine to methionine (fig 2). Folate acts to stabilise the thermolabile enzyme ${ }^{10}$ and serum folate levels above $15.4 \mathrm{nM}$ appear to neutralise the effect of the $677 \mathrm{C}-\mathrm{T}$ mutation and enhance reductase activity. ${ }^{11}$

Hyperhomocysteinaemia has been identified as an independent risk factor for vascular disease. ${ }^{12}{ }^{13}$ It has been shown to be associated with carotid artery stenosis, and predicts mortality in patients with coronary artery disease. Reduction of plasma homocysteine levels has been shown to slow the rate of coronary restenosis after angioplasty. ${ }^{13}$ Hyperhomocysteinaemia has also been recognised as a risk factor for venous thrombosis. ${ }^{12}$ The event preceding vascular occlusion, due to an elevated plasma homocysteine, is endothelial damage and dysfunction. Avascular necrosis of the femur head has also been described in association with hyperhomocysteinaemia. ${ }^{14}$ In addition, this patient had severe osteopenia. This has recently also been linked to hyperhomocysteinaemia ${ }^{15}$ although liver disease per se can lead to hepatic osteodystrophy.

In conclusion, our patient presented with a triad of marked intrahepatic vascular abnormalities with nodular regenerative hyperplasia, portal vein thrombosis, and subsequent avascular necrosis of the femur heads. These vascular phenomena were set in the context of a raised plasma homocysteine. To our knowledge this is the first case of nodular regenerative hyperplasia described in association with hyperhomocysteinaemia.

\section{ACKNOWLEDGEMENTS}

We would like to thank Professors E Legius and G Mathijs for determination of the gene mutation.

\section{Authors' affiliations}

O Buchel, F Nevens, J Fevery, Division of Hepato-biliary and Pancreas Diseases, University Hospital Gasthuisberg, Leuven, Belgium T Roskams, B Van Damme, Department of Pathology, University Hospital Gasthuisberg, Leuven, Belgium

J Pirenne, Division of Abdominal Transplant Surgery, University Hospital Gasthuisberg, Leuven, Belgium

Conflict of interest: None declared.

Correspondence to: Professor J Fevery, University Hospital Gasthuisberg, Leuven, B 3000 Belgium;

Johan.Fevery@uz.kuleuven.ac.be

Revised version received 15 February 2005

Accepted for publication 21 February 2005

\section{REFERENCES}

1 Farrell GC. Nodular regenerative hyperplasia. In: Feldman M, Friedman LS, Sleisinger MH, eds. Sleisinger and Fortran's Gastrointestinal and Liver Disease, 7th edn. Philadelphia: Saunders, 2002:1434.

2 Wanless IR. Micronodular transformation (nodular regenerative hyperplasia) of the liver. A report of 64 cases among 2500 autopsies and a new classification of benign hepatocellular nodules. Hepatology 1990;1 1:787-97.

3 Shimamatsu K, Wanless IR. Role of ischemia in causing apoptosis, atrophy, and nodular hyperplasia in human liver. Hepatology 1997;26:343-50.

4 Sekiya M, Sekigawa I, Hishikawa T, et al. Nodular regenerative hyperplasia of the liver in systemic lupus erythematosus. The relationship with anticardiolipin antibody and lupus anticoagulant. Scand J Rheumatol 1997;26:215-17.

5 Gonzalez-Alvaro I, Carmona-Ortell L, Amigo-Etxenagusia A, et al. Nodular regenerative hyperplasia of the liver and primary Sjogren's syndrome. J Rheumatol 1994:21:168-9.

6 Morla RM, Ramos-Casals M, Garcia-Carrasco M, et al. Nodular regenerative hyperplasia of the liver and antiphospholipid antibodies: report of two cases and review of the literature. Lupus 1999;8:160-3.

7 Levy S, Diebold MD, Nguyen $P$, et al. Nodular regenerative hyperplasia of the liver associated with a factor V Leiden mutation. Eur J Gastroenterol Hepatol 1998; 10:721

8 Han D, Kim S, Chang J, et al. Avascular necrosis following renal transplantation. Transplant Proc 1998;30:3034-5.

9 Reichert-Penetrat S, Trechot P, Barbaud A, et al. Bilateral femoral avascular necrosis in a man with psoriasis: responsibility of topical corticosteroids and role of cyclosporine. Dermatology 2001;203:356-7.

10 Frosst $\mathbf{P}$, Blom HJ, Milos R, et al. A candidate genetic risk factor for vascular disease: a common mutation in methylenetetrahydrofolate reductase. Nat Genet 1995;10:111-13.

11 Jacques PF, Bostom AG, Williams RR, et al. Relation between folate status, a common mutation in methylenetetrahydrofolate reductase, and plasma homocysteine concentrations. Circulation 1996;93:7-9.

12 Pathare A, Alkindi S, Albalushi T, et al. Heterozygous methylene tetrahydrofolate reductase mutation with mild hyperhomocysteinemia associated with deep vein thrombosis. Clin Lab Haematol 2004;26:143-6.

13 Schnyder G, Roffi M, Pin R, et al. Decreased rate of coronary restenosis after lowering of plasma homocysteine levels. N Engl J Med 2001;345: 1593-600

14 Zalavras CG, Malizos KN, Dokou E, et al. The 677C-> T mutation of the methylene-tetra-hydro-folate reductase gene in the pathogenesis of osteonecrosis of the femoral head. Haematologica 2002;87:111-12.

15 van Meurs JB, Dhonukshe-Rutten RA, Pluijm SM, et al. Homocysteine levels and the risk of osteoporotic fracture. N Engl J Med 2004;350:2033-41. 